\title{
HETEROCYCLES AWARD
}

\section{HETEROCYCLES is pleased to announce Heterocycles Award. In recognition of an outstanding oral presentation at the $47^{\text {th }}$ Congress of Heterocyclic Chemistry October 26 to 28, 2017, Kochi Prefectural Culture Hall, Kochi, Japan}

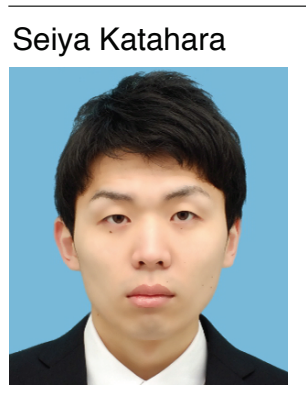

Title: Reductive Approach to Nitrones from $\mathrm{N}$-Siloxyamides and $\mathrm{N}$-Hydroxyamides

Seiya Katahara was born in 1992 in Tokyo, Japan. He is a Ph.D. course student in Center for Molecular Chemistry, School of Fundamental Science and Technology, Keio University working with Prof. Noritaka Chida and Prof. Takaaki Sato. His research interest includes development of nitrogen-containing molecules conversion reactions.

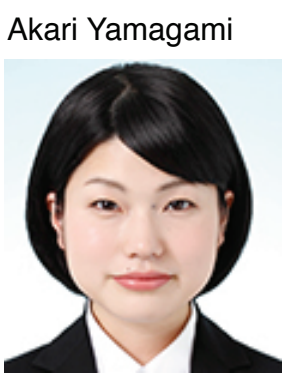

Title: Synthesis and Properties of the New V-Shaped Xanthene Dyes

Akari Yamagami was born in 1994 in Kyoto. She is a second year Master course student in Graduate School of Life and Environmental Sciences, Kyoto Prefectural University, working with Professor Kazunori Tsubaki. Her research interests include synthesis of new types of V-shaped xanthene fluorescent dyes and evaluation in terms of their optical properties. She received a poster award from The Japanese Association for Organic $\pi$-Electron Systems in 2016.

Keita Ashida

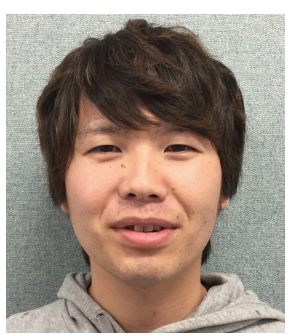

Title: Synthesis of Polycyclic $\gamma$-Lactams by Ni(0)-Catalyzed [2+2+1] Cycloaddition of Imines and Alkenes with Carbon Monoxide

Keita Ashida was born in 1992 in Kyoto. He is a second year Master course student in the Faculty of Engineering, Osaka University, working with Prof. Sensuke Ogoshi. His research interests are focoused on the development of the Ni-catalyzed enantioselective synthesis of heterocyclic compounds via nickelacycle intermediates. 